Original Research

\title{
H-Ras Pre-mRNA Contains A Regulatory Non-coding RNA
}

\author{
Mariette Kokolo, Montse Bach-Elias *
}

Institute of Biomedical Research of Barcelona (IIBB)-Spanish National Research Council (CSIC), Barcelona, Spain; E-Mails: kokolo_mariette@yahoo.fr; montse.bach@iibb.csic.es

*Correspondence: Montse Bach-Elias; E-Mail: montse.bach@iibb.csic.es

Academic Editor: Michael R. Ladomery

Special Issue: Alternative Splicing: A Key Process in Development and Disease

OBM Genetics

2020, volume 4 , issue 4

doi:10.21926/obm.genet.2004118
Received: June 25, 2020

Accepted: October 29, 2020

Published: November 16, 2020

\begin{abstract}
Non-coding RNAs (ncRNAs) have emerged as one of the most abundant regulatory molecules. However, their roles and functions are significantly different from those of proteins. Moreover, around $95 \%$ of the human genome contains non-coding DNA. ncRNAs contribute by far the majority of human transcriptional units, and the functions of the most are yet unknown. Here, we highlight that an important RNA sequence region, encompassing an exonintron hairpin loop (also called IDX-rasISS1), of the H-Ras pre-mRNA may encode an ncRNA that regulates p68 RNA helicase. This observation is remarkable owing to the fact that this helicase is responsible for upregulating the hairpin loop. This indicates that an inhibitory feedback mechanism acting on the p68 RNA helicase is mediated by higher structural levels of the hairpin-loop. Initially, two observations prompted the present study: i) previous results revealed down-regulation of p68 RNA helicase resulting from overexpression of the IDX hairpin loop in HeLa cells, and ii) the secondary structure of the IDX hairpin loop resembles pri-miRNAs, implying that an miRNA could be processed from the hairpin loop-containing primiRNA and regulate 68 RNA helicase. To validate our hypothesis, we directly compared p68 RNA sequences and the hairpin loop in silico. Furthermore, RNAi assays containing the hairpin loop as an miRNA precursor were conducted, using the pTer vector, to explore the effects on 68 RNA helicase expression levels. These RNAi analyses were quantified by Western blots
\end{abstract}

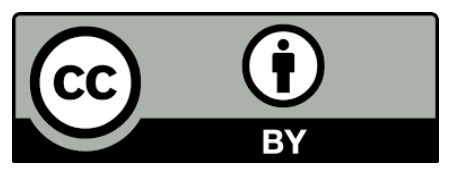

(C) 2020 by the author. This is an open access article distributed under the conditions of the Creative Commons by Attribution License, which permits unrestricted use, distribution, and reproduction in any medium or format, provided the original work is correctly cited. 
(using anti-p68 RNA helicase and anti-EIF2 $\alpha$ ) and Fluc/Rluc 3'UTRs/CDS assays. The effect of hairpin loop overexpression on cell growth and cancer processes was also investigated by analyzing cell-cycle phases and miR-206 expression. Finally, alternative splicing microarrays containing apoptosis targets were incubated to verify whether pre-mRNAs other than $\mathrm{H}$-Ras could also present a similar hairpin loop structure regulated by p68 RNA helicase. We observed that overexpression of the hairpin loop does not activate the phosphorylation of EIF2 $\alpha$ and, therefore, does not activate PKR interferon-induced apoptosis. Moreover, a similar effect on p68 RNA helicase-mediated interference is observed during the upregulation of the hairpin loop. Finally, we also identified a similar hairpin loop-like structure in an alternative splicing region of MAPK12/ERK6. Thus it can be inferred from our findings that the alternative splicing exon IDX from $\mathrm{H}$-Ras, coupled with the immediately downstream intron sequences, may contain an ncRNA. We also unveil one potential function of this ncRNA whose expression is regulated by alternative splicing decisions.

\section{Keywords}

p68 RNA helicase; ncRNA; miRNA; intronic miRNA miR-206, H-Ras; ERK6

\section{Introduction}

Ras genes have been extensively investigated as an important hallmark of normal and tumorigenic cellular signals and natural transduction events [1-3]. The Ras family includes the three genes $\mathrm{c}-\mathrm{H}$-ras, $\mathrm{c}-\mathrm{K}$-Ras, and $\mathrm{c}-\mathrm{N}$-ras. Mutated $\mathrm{H}$-Ras on codon 12 has been accounted as a diagnostic marker for Costello syndrome, a rare, multi-system disease that affects major organs, significantly reducing the quality of life of the afflicted children [4-6]. Two H-Ras [7-9] and two K-Ras [10] mRNAs are produced from the same pre-mRNA by alternative splicing of the 3 '-terminal exons, with the Cterminal protein being strongly affected by the alternative pre-mRNA process in both cases. $\mathrm{H}$-Ras pre-mRNA contains the alternative exon IDX and the inclusion or exclusion of this exon results in the formation of either p19 H-Ras or p21 H-Ras proteins, respectively [7-9]. p19 H-Ras has a distinct and complementary function to p21 H-Ras $[8,9,11,12]$. The most important characteristic of $\mathrm{p} 19$ $\mathrm{H}$-Ras is that it is not localized in the cytoplasmic membrane $[8,9]$. Basically, p19 H-Ras binds to RACK1 and $p 73 \alpha / \beta$, may activate telomerase through $p 73$, regulates the mTOR pathway, and may induce $\mathrm{G} 1 / \mathrm{S}$ delay and a reversible quiescence state, thereby prohibiting entry into apoptosis. Furthermore, p19 H-Ras RNAi increases cell proliferation [8, 9]. Both p19 H-Ras and p21-H-Ras mRNAs are observed in all human tissues tested to date, with most tissues containing $50 \%$ of each $[8,9]$. Earlier results claimed that the Costello mutant P19G12S H-Ras demonstrated a clear specific upregulation of several miRNAs [13].

Previous in vivo and in vitro results from our group characterized both cis-acting regulatory sequences and trans-splicing factors engaged in the regulation of IDX inclusion [14]. We have also highlighted that an intronic silencer sequence (named rasISS1) located downstream of IDX (See Figure 1) is one of the hot sequences involved in the negative regulation of the IDX upstream exon splicing [14]. Indeed, this inhibitory effect seems to be partially mediated by the binding of hnRNP A1, an IDX inclusion inhibitor and, consequently, a p19 H-Ras expression inhibitor. Moreover, p68 
RNA helicase has been documented to be a key regulator of this alternative splicing by inhibiting IDX inclusion and, therefore, p19 H-Ras expression [14]. p68 RNA helicase has been shown to bind to both IDX and rasISS1 sequences through specific RNA columns, and this finding subsequently motivated us to explore its putative secondary structure, obtained using the Mfold software (Figure 1), as a regulatory hallmark for the IDX inclusion/exclusion [14]. This secondary structure reflects a high complementarity between IDX and the immediately downstream D2 intron that includes rasISS1. It should also be noted that this secondary structure traps the 5 '-SS region of intron D2, in competition with U1snRNP binding. Several factors, including SC35, SRp40, and FUS/TLS, have been known to be trans-acting activators of IDX inclusion and, therefore, p19 H-Ras expression [14, 15]. Furthermore, in vivo RNAi-mediated depletion of hnRNP $\mathrm{H}$ has been attributed to reducing $\mathrm{p} 19 \mathrm{H}$ Ras abundance [15]. As expected, the function of all these factors should be interrelated and in this regard, we have previously reported that p68 RNA helicase unwinds the Hairpin-Loop IDX-rasISS1 illustrated in Figure 1 and that this unwinding prevents binding of hnRNP H to IDX-rasISS1 [15].

\section{Hairpin-Loop}

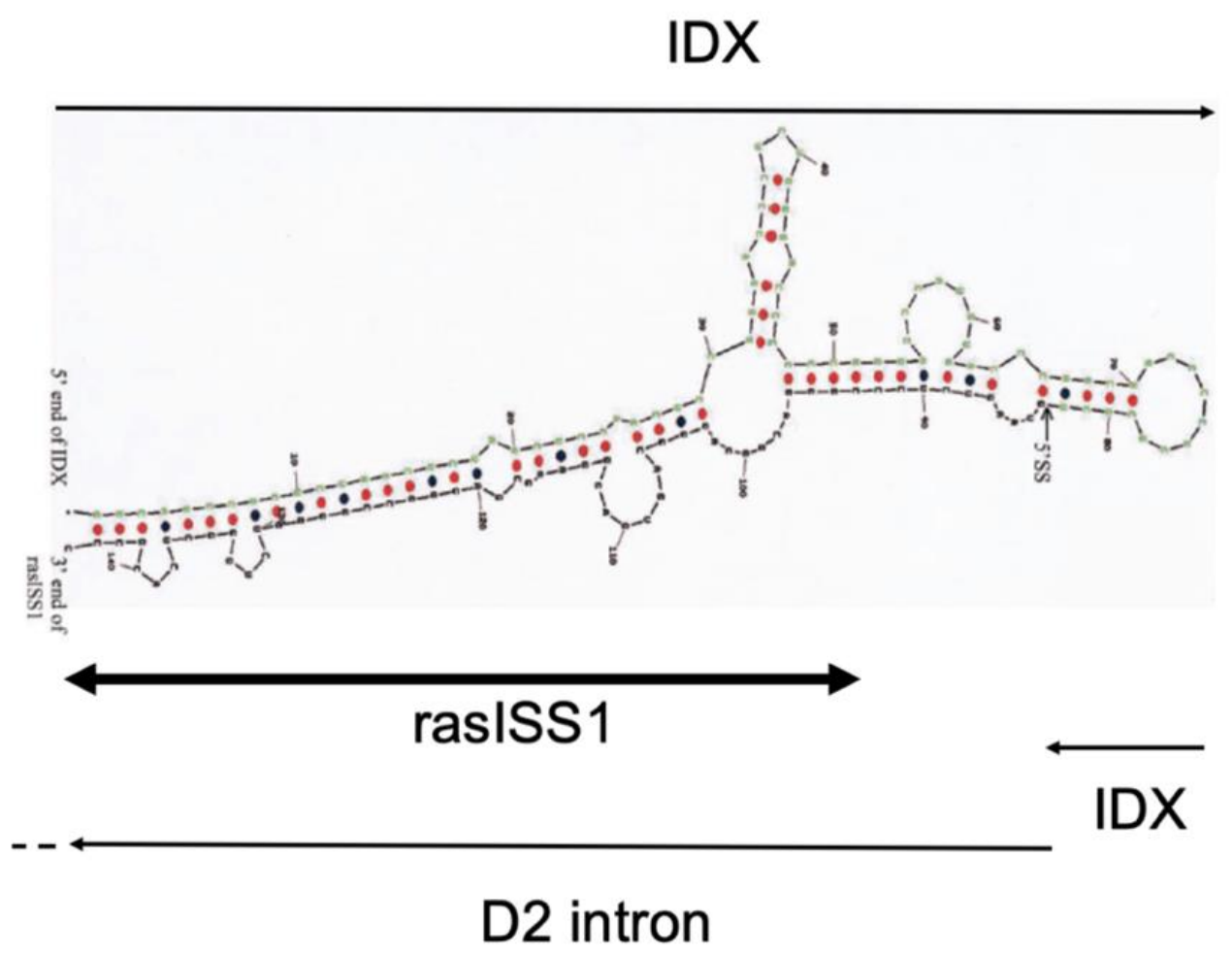

Figure 1 Secondary structure of the Hairpin-Loop comprising the IDX exon plus $3^{\prime}$ intron with rasISS1. IDX is found to extend from the $5^{\prime}$-terminus of the left-hand strand through the green ribonucleotides to 5' SS (splice-site). The $3^{\prime}$ intron of IDX (right-hand strand) extends from $5^{\prime}$ SS through the black ribonucleotides to the $3^{\prime}$-terminus of rasISS1. This structure has been published [14] and was derived with the help of the Mfold software (http://unafold.rna.albany.edu/?q=mfold).

p68 RNA helicase belongs to the family of DEAD-box proteins, all of which have a characteristic Asp-Glu-Ala-Asp (DEAD) box as one of eight highly conserved sequence motifs and are involved in ATP hydrolysis [16]. Furthermore, research has highlighted the involvement of RNA helicases in 
ribosome biogenesis, splicing and alternative splicing, mRNA export from the nucleus, RNA decay, and translation initiation $[17,18]$. Several previous studies reported the upregulation of p68 and p72 in colorectal, prostate, and breast cancer [19-22], thereby suggesting the crucial role of helicase in several cancerous processes $[23,24]$. We previously demonstrated the activation of a viability pathway encompassing mTOR and MDM2 Signals mediated by the downregulation of p68 RNA Helicase [25].

Several studies have reported the presence of both small and long non-coding RNAs (ncRNAs) or mRNA-like transcripts in a huge proportion of the mammalian genome [26-30]. Rodriguez et al., for example, claimed that half of all known mammalian miRNAs are inside introns of either proteincoding or non-coding transcription units and that $10 \%$ of miRNAs are encoded by exons of long nonprotein-coding transcripts [31]. These observations resulted in the classification of the majority of miRNAs as intronic or exonic hosted transcription units. Furthermore, alternative splicing signifies that miRNAs can be located in either an exon or an intron depending on the alternative splicing decisions of the host transcript [31,32]. Research has validated the role of all these ncRNAs in the regulation of cell-cycle pathways [26-30].

Herein we emphasized the important region that regulates the alternative splicing of $\mathrm{H}$-Ras, namely, the Hairpin-Loop IDX-rasISS1, may also contain a new ncRNA that downregulates p68 RNA helicase, which is itself responsible for the up-expression of this Hairpin-Loop, in a feedback-type mechanism.

\section{Methods}

\subsection{Cell Cultures, Plasmids, and Transfections}

HeLa cell culture was described previously [8]. The isolation of clone 13 was detailed elsewhere [25]. In brief, stable HeLa cells (clone 13) containing the p68 RNA helicase RNAi induced by doxycycline (DOX) were obtained by co-transfecting pTER-p68 RNA helicase RNAi/pCDNA 6 /TR. The selection was performed with zeocine/blasticidine $100 / 5 \mu \mathrm{g} / \mathrm{mL}$ and RNAi induced by the addition of $2 \mu \mathrm{g} / \mathrm{mL}$ DOX, resulting in $70-80 \%$ downregulation of p68 RNA helicase. Transient transfections with pSuper and pSuper-p68 RNA helicase RNAi were described previously [14]. The pTer-HairpinLoop plasmid was obtained by amplifying the sequence shown in Figure 2 with specific 5 '-terminal and $3^{\prime}$-terminal deoxyoligonucleotides and cloned in pTer vector as directed by the manufacturer. psiCheck-68 RNA helicase 3' UTR and psiCheck-p68-[421-586] CDS plasmids were obtained by amplifying the sequence given in Figure 2 with specific $5^{\prime}$-terminal and $3^{\prime}$-terminal deoxyoligonucleotides. Three independent experiments were conducted, each per triplicate. 
A)

P68 RNA helicase

Hairpin-loop

P68 RNA helicase

Hairpin-loop

P68 RNA helicase

Hairpin-loop

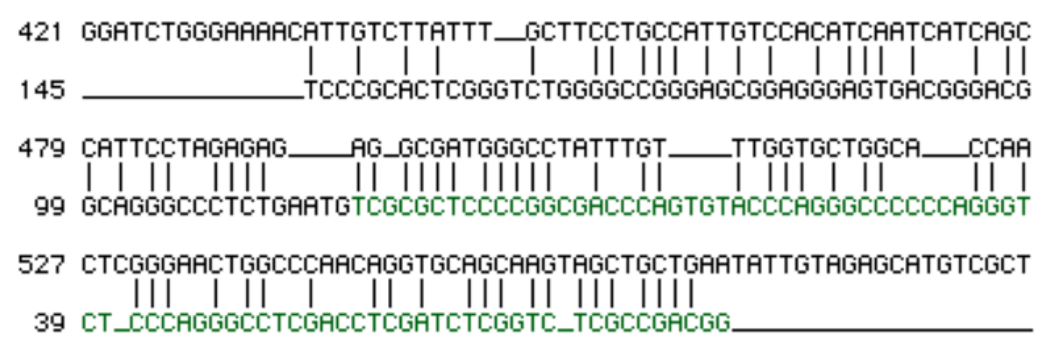

B)

3' UTR-P68 RNA helicase IDX

3' UTR-P68 RNA helicase IDX

3' UTR-P68 RNA helicase
181 TTRGGTTTTTTTTGTACTAGARAATGC_AGG_CAGTGTTTCACARARGT

82 I |||||| | | || ||| ||

229 AARTG_TACAGTGATTTGARATACARTARTGAAGGCAATGCATGGCCTTCCAR 47 CCCAGGGTCTCCCAGGGC_CTCGACCTC_G_AT_CT_CGGTCTCG_CCG_ACGG 281 TARARARTATTTGRAGACTGRARRARARAR

C)

3' UTR-P68 RNA helicase rasISS1

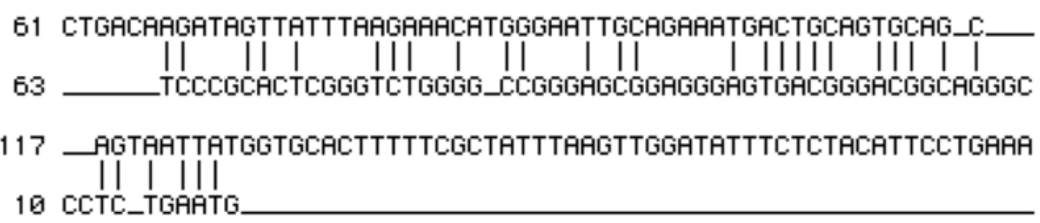

3' UTR-P68 RNA helicase rasISS1

Figure 2 Sequence comparisons between the Hairpin-Loop and p68 RNA helicase mRNA. IDX is represented in green, whereas the rasISS1-containing sequence in black. A) A direct comparison of the p68 RNA helicase sequence and the Hairpin-Loop. The best match was detected between nucleotides [421-586] of p68 RNA helicase mRNA CDS. B) A direct comparison between the p68 RNA helicase mRNA 3'-UTR and the IDX sequence only. The 3'-terminus of this 3'-UTR portrayed the best matches. C) A direct comparison between the p68 RNA helicase mRNA 3'-UTR and the rasISS1-containing sequence only. The best matches were observed in the middle of this $3^{\prime}$-UTR. Comparisons were performed exploiting the MacMolly Align program.

\subsection{Antibodies}

The following antibodies were used: anti-GAPDH (Abcam); anti-p-EIF2 $\alpha$ (Biosource); anti-p68 RNA helicase (Santa-Cruz Biotechnology) and SP1 anti-p19-HRas [8].

\section{3 mir-206 Taqman Real-Time}

MicroRNA Reverse Transcriptase Reaction: cDNA was generated by reverse transcription from enriched miRNA samples (miRVANA kit) using specific miRNA primers from the Taq Man MicroRNA Assay and reagents from the Taq Man MicroRNA Reverse Transcriptase kit (AB Applied Biosystems) following the manufacturer's instructions. Briefly, $1.33 \mu \mathrm{L}$ of each resulting cDNA was amplified by PCR (Polymerase Chain Reaction) using Taq Man MicroRNA Assay primers with Taq Man Universal Non amperase PCR Master Mix (in a total volume of $20 \mu \mathrm{L}$ ) and analyzed with a $7500 \mathrm{ABI}$ PRISM 
Sequence Detector System as per the instructions provided by the manufacturer. mRNA expression was calculated from the relevant signals by normalization with respect to the signal for U6.

Quantitative reverse transcription-PCR of miRNAs: Stem-loop quantitative reverse transcriptionPCR (RT-PCR) for mature miRNAs was performed as described previously with the help of an Applied Biosystems.

ABI 7000 Real-Time PCR system: All PCR reactions were run in triplicate and gene expression, relative to $U 6$, was calculated using the $2-\Delta \Delta C$ t method [33]. Three independent experiments were done, each per triplicate.

\subsection{Determination of Cell-Cycle Phase Percentages}

HeLa cells were transfected with different plasmid DNAs encoding a GFP sequence, as described previously [8, 14]. Cells were incubated with $1 \mu \mathrm{g} / \mathrm{mL}$ Hoechst 33342 for $1 \mathrm{~h}$ at $37^{\circ} \mathrm{C}$, then washed, and resuspended at $1 \times 10^{6}$ cells/mL in DMEM (Dulbecco's Modified Eagle Medium) supplemented with 10\% FBS (Fetal Bovine Serum), penicillin-streptomycin, and Hoechst 33342 prior to analysis. Flow cytometric experiments were executed using a MoFlo flow cytometer (DakoCytomation, Fort Collins, Colorado, USA). The samples were excited with the blue line of a Coherent Enterprise II argon-ion laser (488 $\mathrm{nm}$ ), facilitating the acquisition of forward scatter (FS), side scatter (SS), and green $(530 \mathrm{~nm})$ fluorescence from GFP. UV emission (20 W) was used to excite Ho33342 blue fluorescence $(450 \mathrm{~nm})$. Doublets were discriminated using an integral/peak dot plot of Hoechst fluorescence. The optical alignment was based on the optimized signal from $10 \mu \mathrm{m}$ fluorescent beads (Flowcheck, Coulter Corporation, Miami, Florida, USA).

\subsection{Rluc/Fluc Assay}

This assay was performed using a Dual-Glo ${ }^{\mathrm{TM}}$ Luciferase Assay System, according to the manufacturer's directions, as previously detailed [25].

\subsection{Alternative Splicing Microarray}

The alternative splicing array (Exonhit) with apoptosis-related genes was from Agilent custom AMADID $44 \mathrm{k}$. Incubations were performed following the manufacturer's instructions, as described previously. HeLa cells were transiently transfected with pSuper-p68 RNA helicase RNAi and compared with HeLa transiently transfected with pSuper empty vector. All experiments were performed in triplicate with either pSuper-p68 RNAi-Cys5xpSuper-Cy3 or pSuper-p68 RNAiCys3xpSuper-Cy5. The microarray also contained duplicate samples of the same gene.

\subsection{Other Methods}

As described previously [34], the protein was extracted for SDS (Sodium Dodecyl Sulfate) gels and Western blots. However, for the protein to be assayed with anti-pEIF2 $\alpha$ antibody, the extracts were prepared using RIPA buffer (10 mM Tris- $\mathrm{HCl}$, pH 8.0, $140 \mathrm{mM} \mathrm{NaCl}, 1$ mM EDTA, 0.5 mM EGTA, $1 \%$ Triton X-100, $0.1 \%$ sodium deoxycholate, $0.1 \%$ sodium dodecyl sulfate, Aprotinin, Leupeptin, $200 \mathrm{nM}$ sodium orthovanadate, $50 \mathrm{mM} \mathrm{NaF}$ ). Chemiluminescence reaction was detected for $5 \mathrm{~min}$ in LAS-4000 Image analyzer (GE Healthcare Life Sciences, Pittsburgh, PA) and quantified using Fujifilm Multi Gauge V3.0 imaging software (GE Healthcare Life Sciences). 


\section{Results}

\subsection{The IDX-rasIS1 Hairpin-Loop Shows a Secondary Structure Likely to Contain miRNAs}

An important regulation hallmark is located between exon 3 and exon $4 A$ of the $H$-Ras pre-mRNA. Moreover, the presence of an alternative exon (IDX) allows the synthesis of two alternative proteins, namely p21 H-Ras and p19-H-Ras, which indulge in distinct and complementary cellular roles [8, 9]. This alternative splicing is regulated by the p68 RNA helicase, an important splicing factor, by winding/unwinding a secondary structure of the IDX-rasISS1 Hairpin-Loop (Figure 1). Several previous observations and new findings prompted us to investigate the role of this Hairpin-Loop structure itself in the cell: a) the balance between p19/p21 H-Ras is almost 50\% [8] in many tissues and this finding indicated that $50 \%$ of the alternative exon IDX, which is spliced out, binds to the two D1 and D2 adjacent introns [8]; b) this Hairpin-Loop renders a stable secondary structure in native gels [15]; c) the Hairpin-Loop binds and is unwound by p68 RNA helicase [15] and p68 helicase downregulation increases the amount of IDX containing pre-mRNA, thereby upregulating $\mathrm{p} 19-\mathrm{H}-$ Ras [14]; d) the Hairpin-Loop has been stably identified in splicing denatured gels, and Hairpin-Loop containing-lariats were sequenced from such gels and e) it is very conserved in different mammalian cells $[8,14,15]$. However, the presence of the Hairpin-Loop in cytoplasmic DNA-depleted RNA fraction was inconclusive, mainly due to its minimal level. Nonetheless, we did obtain an in vivo chimera between a 60 mer region of the Hairpin-Loop and t-RNA, in the presence of selected labeled isotopes. RMN preliminary results of this labeled-chimera elucidated $30 \%$ of the 60 mer secondary structure described here (our unpublished results).

We observed new findings while performing some control experiments. We realized that the overexpression of the Hairpin-Loop RNA reduced the amount of p68 RNA helicase (see below). This observation, in addition to the secondary structure as shown in Figure 1, which resembles the secondary structures of pre-miRNAs, suggested that a miRNA could be processed from the HairpinLoop. To further validate this hypothesis, we executed in silico direct analysis between Hairpin-Loop and p68 RNA helicase mRNA sequences. Figure 2 illustrates several sequences of the Hairpin-Loop base-pairs and their mismatches, with an internal region of the p68 RNA helicase mRNA CDS (bps 481-586), as well as a sequence of IDX-only base-pairs and mismatches, with the 3 '-terminus of the p68 RNA helicase 3'-UTR and rasISS1 with the region 61-126 of the p68 RNA helicase 3'-UTR, both also with mismatches. These observations denoted that the Hairpin-Loop has a secondary structure, which might act as a substrate of Drosha/Dicer along with putative sequences that are suggestive of miRNAs with p68 RNA helicase as a target.

We further checked in silico whether the Hairpin-Loop could present base-pairing with other mRNAs and results are summarized in Supplementary Material 1.

\subsection{The IDX-rasISS1Hairpin Loop Downregulates p68 RNA Helicase}

With the aim to study the cellular role of the Hairpin-Loop, specific assays were conducted to determine the effect of Hairpin-Loop expression in human HeLa cells. Previously it was elucidated that the Hairpin-Loop is a substrate for p68 RNA helicase [15]. Therefore, it is highly likely that overexpression of this sequence should hijack p68 RNA helicase activity (see below). However, if a putative miRNA was located inside the Hairpin-Loop, a decrease in the p68 RNA helicase level should also be perceived. To analyze these hypotheses, the sequence shown in Figure 1 was cloned into 
the RNA expression vector pTer and transiently transfected into the human HeLa clone 13, containing a stable RNAi to p68 RNA helicase mRNA activated by DOX. As is evident from Figure 3A, the Hairpin-Loop downregulates p68 RNA helicase protein levels either in wt HeLa cells (compare lanes 3 and 4, with 50\% downregulation) or in Clone 13 combination with DOX, which also induces the RNAi of p68 RNA helicase (compare lanes 1 to 2, and 5 to 6). Furthermore, samples 1 and 5 also indicate that Hairpin-Loop overexpression enhances the decrease of p68 RNA helicase in DOX inducible cells. Henceforth these results prominently claimed that the Hairpin-Loop directly or indirectly regulates p68 RNA helicase levels.
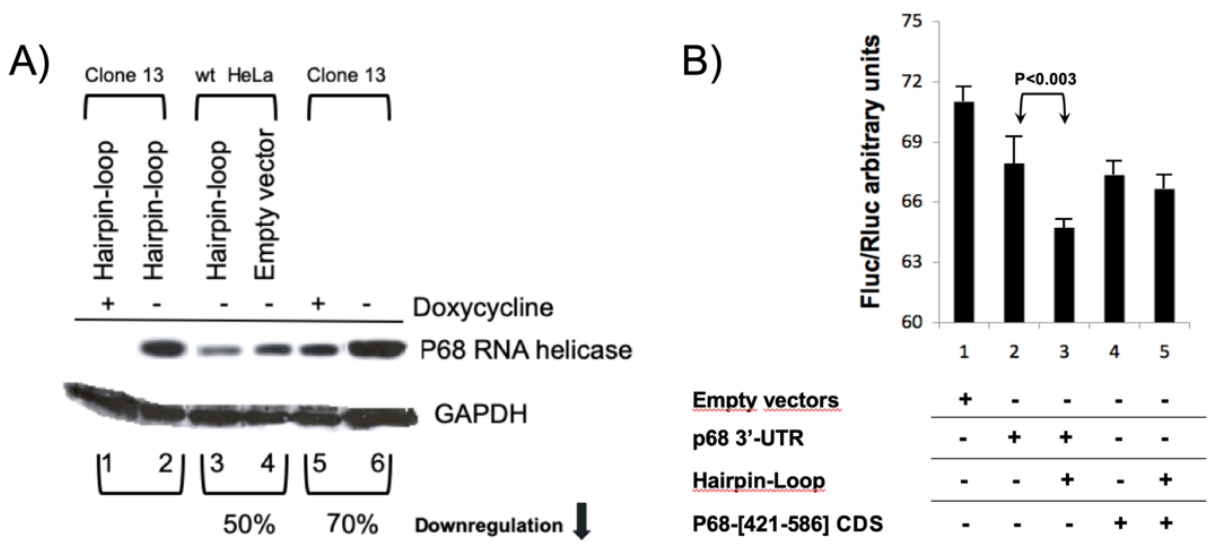

Figure 3 Downregulation of p68 RNA helicase mediated by Hairpin-Loop RNA sequence overexpression. A) Stable clone 13 with a DOX-inducible p68 RNA helicase RNAi (lanes 1,2, 5, and 6), or wt HeLa cells (lanes 3 and 4) were transiently transfected with pSuper.neo.gfp-Hairpin-Loop (Hairpin-Loop) or with pSuper.neo.gfp (empty vector). RNAi of p68 RNA helicase was induced with $2 \mu \mathrm{g} / \mathrm{mL}$ DOX (+ lanes). Protein extracts, obtained after harvesting the cells, were analyzed by Western blot, incubating with antip68 RNA helicase along with the internal control anti-GAPDH. The results were normalized with regards to GAPDH as an internal control. p68 RNA helicase was downregulated by around $70-80 \%$ owing to DOX concentration of $2 \mu \mathrm{g} / \mathrm{mL}$ (compare lanes 5 and 6). Lane 3 reflected a $50 \%$ of p68 RNA helicase downregulation compared to lane 4. B) Wild-type HeLa cells were transiently transfected with both psiCheck and pTer empty vectors (column 1); psiCheck-p68 RNA helicase 3' UTR (column 2); both psiCheck68 RNA helicase 3'-UTR and pTer-Hairpin-Loop (column 3); psiCheck-p68-[421-586] CDS (column 4); and both psiCheck-p68-[421-586] CDS and pTer-Hairpin-Loop (column 5); $\mathrm{P}<0.003$ by Student's $t$-test. Chemiluminescence reaction was detected for $5 \mathrm{~min}$ and quantified. Three independent experiments were performed, each per triplicate.

To further determine whether the Hairpin-Loop exerts its action on p68 RNA helicase by binding directly to the 421-586 CDS region or the 3'-UTR of the p68 RNA helicase mRNA, clones of both these sequences were introduced separately into the Rluc/Fluc reporter vector psiChek2. Figure 3B confirms that overexpression of the Hairpin-Loop in HeLa cells results in RNAi on the p68 RNA helicase 3'-UTR sequence (compare columns 2 and 3) but not the p68 RNA helicase 421-586 CDS (compare lanes 4 and 5). Taken together, these observations were in line with the hypothesis that the exon(IDX)-intron sequence on the Hairpin-Loop may contain one or more ncRNAs that interfere with the p68 RNA helicase 3'-UTR, whose protein is an inhibitor of IDX inclusion. Additionally, these 
findings revealed a feedback-type mechanism in which the high abundance of IDX exclusion, which is the result of a large amount of the IDX host Hairpin-Loop, downregulates p68 RNA helicase, in turn, enhancing this IDX exclusion.

\subsection{The Hairpin-Loop Provokes G1/S Delay but No Apoptosis}

As witnessed above, Hairpin-Loop overexpression in HeLa cells leads to the downregulation of p68 RNA helicase. As reported previously, p68 RNA helicase downregulation (obtained by RNAi) results in a G1/S delay, less cell growth [15, 25], and increased p19 H-Ras protein levels [14], but fails to induce apoptosis [15]. Therefore, we were motivated to explore whether Hairpin-Loop overexpression in HeLa cells would exert a similar effect to that obtained by RNAi-induced downregulation of p68 RNA helicase. Figures 4A and 4B substantiate the fact that Hairpin-Loop overexpression in wild-type HeLa cells, parallel to that for p68 RNA helicase RNAi, also indulges a G1/S delay and decreases the number of apoptotic cells (as portrayed by the apoptotic peak in Figure $4 \mathrm{~A}$ and $4 \mathrm{~B})$.

A)
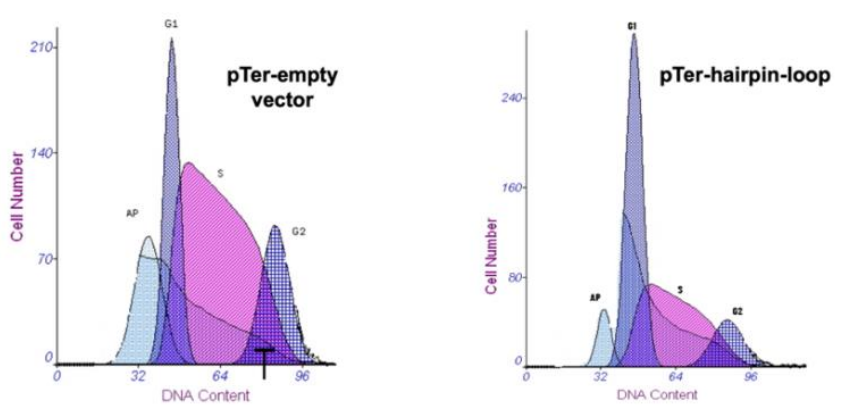

B)

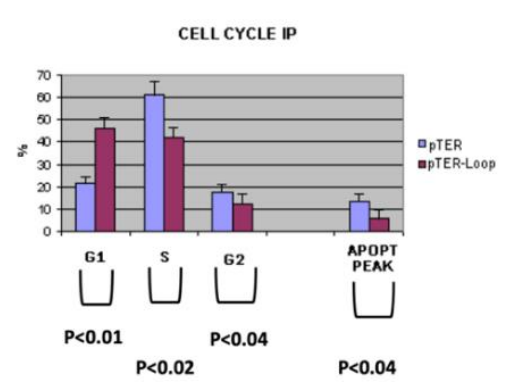

C)

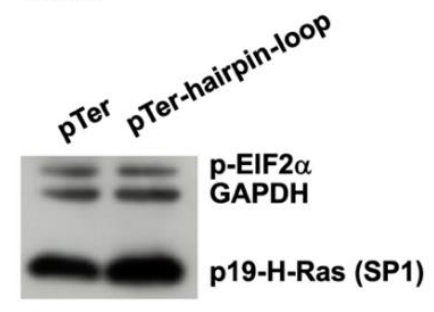

Figure 4 Overexpression of the Hairpin-Loop RNA sequence in wild-type HeLa cells leads to $\mathrm{G} 1 / \mathrm{S}$ delay but reduces apoptosis. A) Cell-cycle phase analysis by FACS (fluorescentactivated cell sorting) in wild-type HeLa cells transiently overexpressing pTer empty vector or pTer-Hairpin-Loop. A representative flow cytometry histogram is provided. B) $\mathrm{G} 1, \mathrm{~S}$, and $\mathrm{G} 2$ percentages are detailed in the graphic. $\mathrm{G} 1$ columns $\mathrm{P}<0.01, \mathrm{~S}$ columns $\mathrm{P}<0.02$, and $\mathrm{G} 2$ columns $\mathrm{P}<0.04$ by Student's $t$-test. The graphic also reveals that the apoptotic peak (APOPT PEAK, $\mathrm{P}<0.04$ ) diminishes in the cells overexpressing the HairpinLoop. C) HeLa cells transiently overexpressing both vectors were harvested and protein extracts obtained. Western blot was employed to analyze the protein content with the apoptotic marker anti-phosphorylated-EIF2 $\alpha$, anti-GAPDH (internal control), and antip19-H-Ras (40\% increase on lane pTer-hairpin-loop). Three independent experiments were done, each per triplicate. 
Double-stranded RNA (dsRNA) sequences similar to those documented in Figure 1 can be detected as foreign to human cells. Such dsRNAs may induce the interferon-inducible gene dsRNAactivated protein kinase (PKR), which acts as an important inhibitor of viral invasion by inhibiting protein synthesis and inducing apoptosis [35, 36]. PKR-mediated impairment of translation initiation is attributed to the phosphorylation of the $\alpha$-subunit of the eukaryotic initiation factor 2 (EIF2 $\alpha$ ) at Ser $51[37,38]$. Thus, EIF2 $\alpha$ kinase acts as a mediator between the transcriptional and translational pathways that regulate vital biological processes such as viral infection and cell proliferation, transformation, and apoptosis. In order to rule out that this defense mechanism against viral infection is activated during Hairpin-Loop overexpression, we harvested wild-type HeLa cells transfected with pTer-Hairpin-Loop and analyzed the extracts, obtained from them, for the phosphorylation of EIF $2 \alpha$ by Western blot. As is evident from Figure 4C, EIF $2 \alpha$ is not phosphorylated; therefore, the interferon-induced defense mechanism PKR/EIF2 $\alpha$ is not activated during overexpression of the Hairpin-Loop. The Hairpin-Loop can thus be concluded as an endogenous RNA rather than a foreign viral RNA. Figure $4 \mathrm{C}$ also claimed a $40 \%$ increase of $\mathrm{p} 19-\mathrm{H}-$ Ras protein with Hairpin-Loop overexpression (lane pTer-hairpin-loop).

In order to ascertain whether overexpression of the Hairpin-Loop provokes similar effects to $p 68$ RNA helicase downregulation by RNAi, we analyzed the percentages of the different cell-cycle phases ensuing from both treatments. As shown in Figure 5, both treatments have a comparable impact on the cell cycle, namely, G1/S delay and reduced $\mathrm{G} 2$ phase.

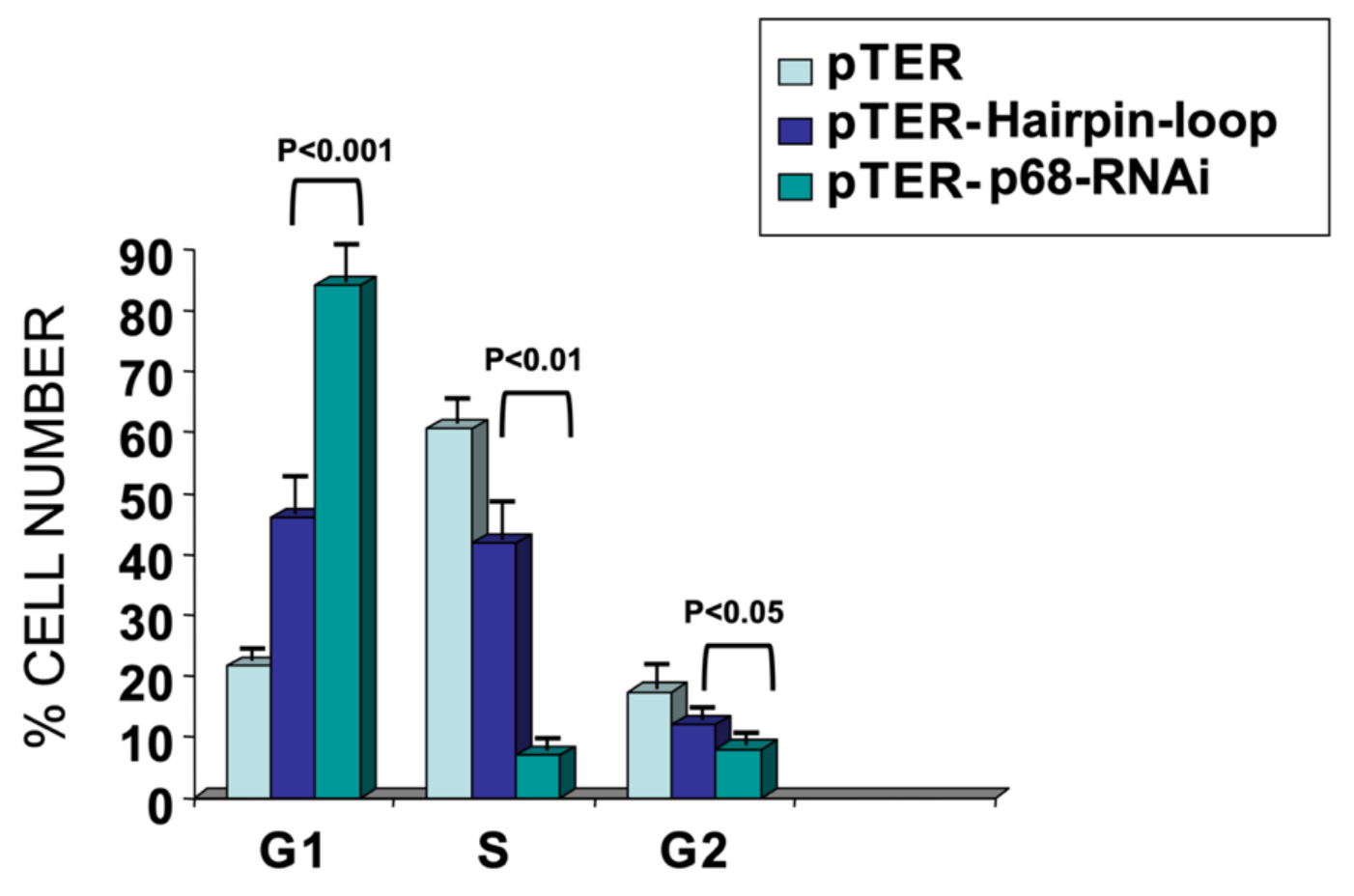

Figure 5 RNAi of the p68 RNA helicase and overexpression of the hairpin-stem loop have a comparable impact on the cell-cycle phases. Cell-cycle phase analysis by FACS (fluorescent-activated cell sorting) in wild-type HeLa cells transiently overexpressing pTER empty vector or pTer-Hairpin-Loop and pTer-p68 RNA helicase RNAi. G1, S, and G2 percentages are detailed in the graphic. $G 1$ columns $P<0.001$, $S$ columns $P<0.01$, and $G 2$ columns $\mathrm{P}<0.05$ (Student's $t$-test). Three independent experiments were done, each per triplicate. 
As clearly demonstrated above (see Figure 3), overexpression of the Hairpin-Loop induces p68 RNA helicase downregulation. Reduced p68 RNA helicase protein level results in the upregulation of certain miRNAs (our unpublished results). Previous research has authenticated the p19 H-Rasinduced upregulation of miR-206 [13], and mir-206 has also been reported to significantly diminish the ability of CN34-LM1 and CN34-BoM1 cells to metastasize to the lung $[39,40]$. To estimate if overexpression of the Hairpin-Loop has the same effect on miR-206, similar transfection assays with pSuper-Hairpin-Loop or pSuper empty vector in wild-type HeLa cells were conducted, and the level of mature miR-206 obtained was analyzed. Figure 6 illuminates that overexpression of the HairpinLoop is responsible for the upregulation of miR-206, and this effect is strongly enhanced when combined with RNAi of the p68 RNA helicase.

\section{$\underline{\operatorname{miR}-206}$}

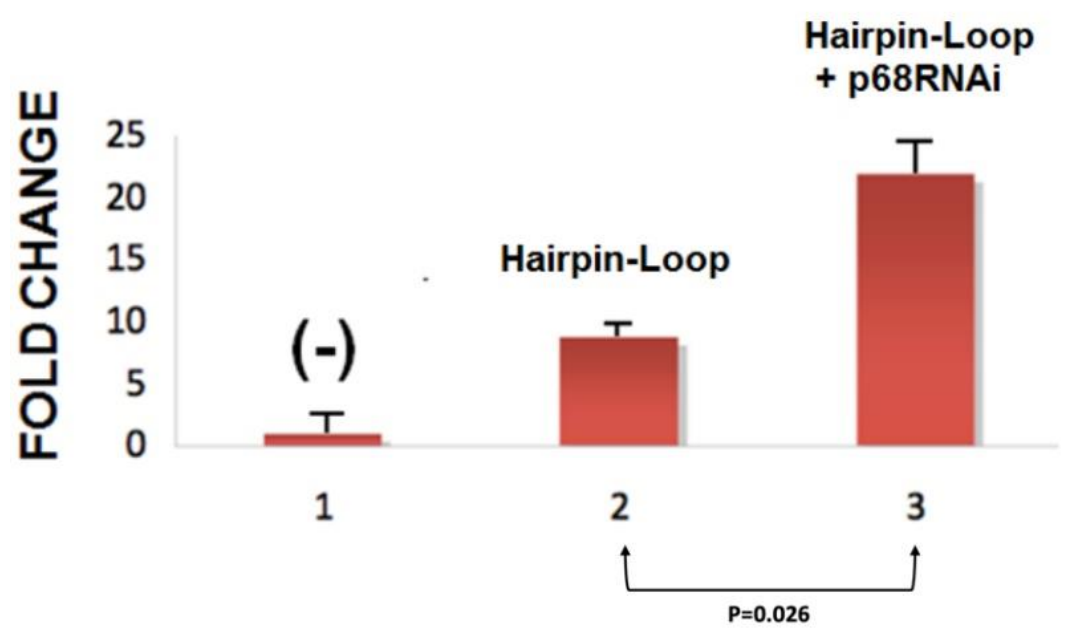

Figure 6 Upregulation of miR-206 mediated by Hairpin-Loop overexpression. Mature mir-206 levels in HeLa and transiently transfected HeLa cells were investigated using Real-time Taqman assays. Lane 1: transfected with pSuper.neo.gfp (empty vector); lane 2: transfected with pSuper.neo.gfp-Hairpin-Loop; lane 3: co-transfected with pSuper.neo.gfp-Hairpin-Loop and pSuper-p68 RNA helicase RNAi (Student's $t$-test, $P=0.026$ comparing lane 2 and 3 ). Three independent experiments were done, each of them per triplicate.

Thus to summarize, Hairpin-Loop overexpression provokes downregulation of p68 RNA helicase, which has the same effect as p68 RNA helicase RNAi: no apoptosis, G1/S delay, and upregulation of miR-206.

\subsection{MAPK12/ERK6 Alternative Exon 8 and Its Downstream Intron Show a Hairpin-Loop Structure Similar to IDX-rasISS1 Hairpin-Loop}

Finally, we hypothesized whether pre-mRNAs other than $\mathrm{H}$-Ras could also exhibit a similar Hairpin-Loop structure regulated by the p68 RNA helicase. One approach to address this question involves comparing the Hairpin-Loop with human genomic sequences and relaxing the number of mismatches. Unfortunately, the results of this study were not conclusive. Subsequently, we decided 
to analyze human alternative splicing exon arrays (Agilent apoptosis Exonhit custom AMADID $44 \mathrm{k}$ ) with mRNA from HeLa cells transfected with pSuper-p68 RNA helicase RNAi and compare with cells transfected with pSuper (empty vector). The results of this study have been submitted in the GEO bank with accession number GSE20010. Table 1 details the results with two selected proteins, namely, MDM2 and MAPK12/ERK6, both of which exhibit exon skipping after RNAi of the p68 RNA helicase. MDM2 is an important apoptosis factor regulating the p53 pro-apoptotic factor [41], whereas MAPK12/ERK6 appears to function as a signal transducer during the differentiation of myoblasts into myotubes [42]. We then examined the RNA folding of the sequences of these two genes that contain the alternative exon plus sequences of the $3^{\prime}$-downstream intron (in analogy with the IDX-rasISS1 Hairpin-Loop). Surprisingly, we observed that the 3 ' intron downstream of the alternative exon 8 (E8) from MAPK12/ERK6 has 60\% homology with rasISS1 (see Figure $7 \mathrm{C}$ ). It was also found that one of the Hairpin-Loops obtained using the Mfold software presents a secondary structure with close resemblance to the IDX-rasISS1 Hairpin-Loop (compare panels B and D in Figure 7). Conversely, similar features were absent in the MDM2 alternative exon 7. We, therefore, propose that these two genes are simultaneously regulated by p68 RNA helicase through a winding/unwinding activity on a similar Hairpin-Loop structure containing alternative exons that could be spliced out as complete introns.

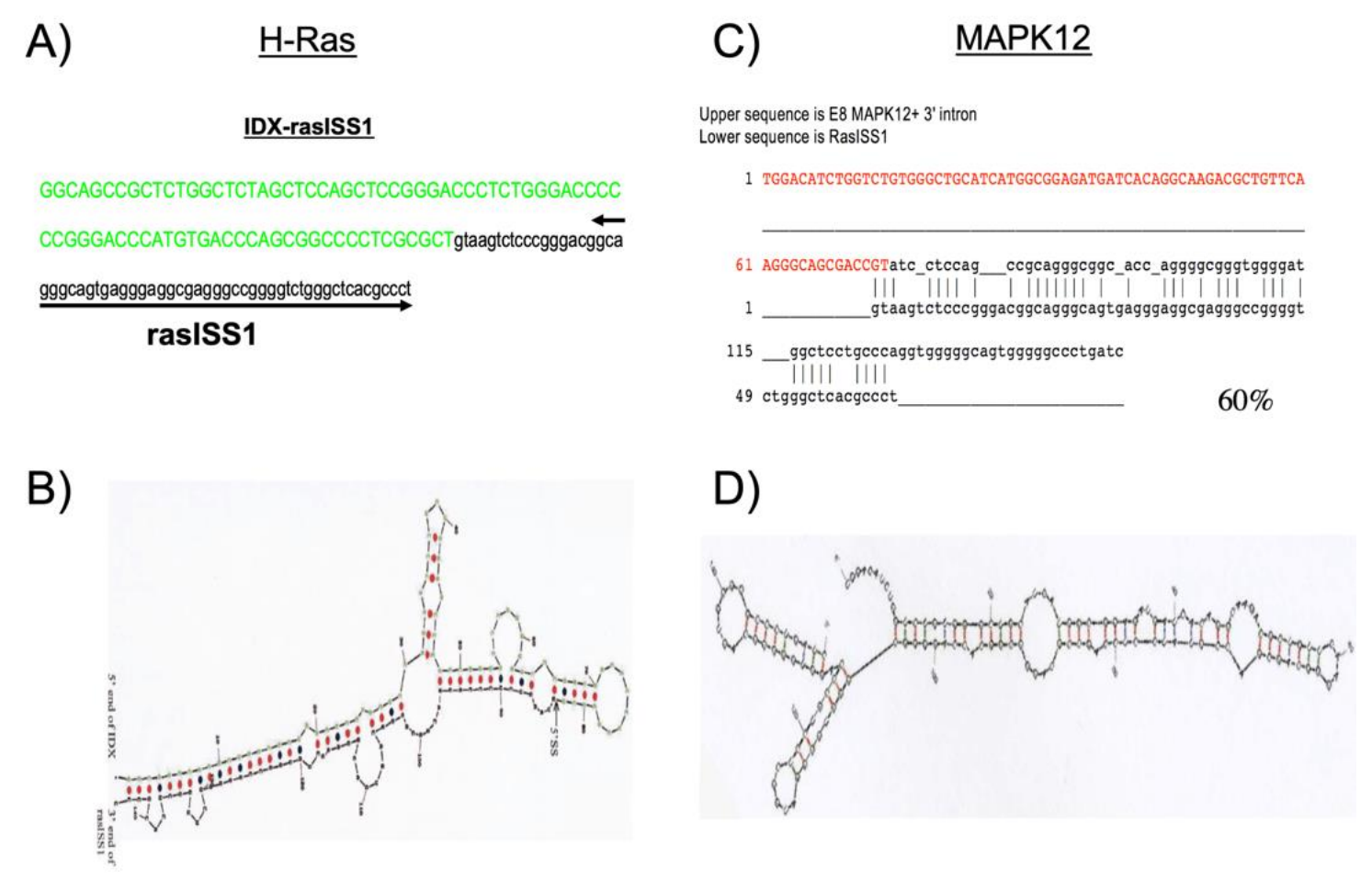

Figure 7 Comparison between H-Ras and MPK12 sequences and secondary structures. A) The sequence of the IDX H-Ras exon (in green) and the $3^{\prime}$ downstream intron (in black) containing the rasISS1. B) Secondary structure of the sequence given in A), as folded by the Mfold program. C) A comparison of the sequence of the E8 MAPK12/ERK6 exon (in red) and the 3' downstream intron (in black) with the IDX 3' downstream intron (in black, encompassing the gtaagt......gccct region). These two intronic sequences exhibit $60 \%$ homology. D) The secondary structure of the MAPK12/ERK6 sequence provided in C), as folded by the Mfold program, resembles the secondary structure outlined in panel B). 
Table 1 Selected results for the alternative splicing array Agilent custom AMADID 44 k (Exonhit) with apoptosis-related genes. HeLa cells transiently transfected with pSuper-p68 RNA helicase RNAi were compared with wild-type HeLa transiently transfected with pSuper empty vector. The experiments were conducted in triplicate (A-C columns) with either pSuper-p68 RNAi-Cys5xpSuper-Cy3 (column 1) or pSuperp68 RNAi-Cys3xpSuper-Cy5 (column 2). The microarray also contained duplicate samples of the same gene, as illustrated in duplicated rows. Exon ID denotes the exon identification number according to gene databanks. Alternative exon is the name of the exon as per the gene databanks. A skipped exon was identified when RNAi of the p68 RNA helicase was performed in both cases. Fold-change indicates the upregulation of the exon skipped after p68 RNA helicase RNAi as compared with the empty vector. Wilcoxon rank sum was used to detect significant differences ( ${ }^{* *} \mathrm{P}<0.01$ in columns $\mathrm{A} 1, \mathrm{~B} 1$, and $\mathrm{C} 1 ;{ }^{* * *} \mathrm{P}>0.001$ in columns $\mathrm{A} 2, \mathrm{~B} 2$, and $\mathrm{C} 2$ ).

\begin{tabular}{|c|c|c|c|c|c|c|c|c|c|}
\hline & & & & $\mathbf{A 1}$ & A2 & B1 & B2 & C1 & $\mathrm{C2}$ \\
\hline EXON ID & Gene name & $\begin{array}{l}\text { Alternative } \\
\text { exon }\end{array}$ & Consequence & \multicolumn{6}{|c|}{ FOLD CHANCE } \\
\hline 0180.025.002_B & $\begin{array}{l}\text { Mdm2_transformed_3T3_cell_double_minute_2p53 } \\
\text { _binding_protein_(mouse)_transcript_variant_MDM } \\
\text { 2_XNO_1556 }\end{array}$ & B & exon_skipped & 1.24 & 1.96 & 1.24 & 2.01 & 1.18 & 1.34 \\
\hline 0180.025.002_B & $\begin{array}{l}\text { Mdm2_transformed_3T3_cell_double_minute_2p53 } \\
\text { _binding_protein_(mouse)_transcript_variant_MDM } \\
\text { 2_XNO_1556 }\end{array}$ & B & exon_skipped & 1.28 & 1.72 & 1.31 & 1.80 & 1.05 & 1.31 \\
\hline 0226.035.001_E & Mitogen-activated_protein_kinase_12_XNO_529 & $\mathrm{E}$ & exon_skipped & 1.61 & 1.71 & 1.05 & 1.55 & 1.18 & 1.30 \\
\hline 0226.035.001_E & Mitogen-activated_protein_kinase_12_XNO_529 & $E$ & exon_skipped & 1.54 & 1.59 & 1.15 & 1.35 & 1.18 & 1.31 \\
\hline
\end{tabular}




\section{Discussion}

Non-coding DNA comprises of nearly $97 \%$ of the human genome, with introns embracing the largest proportion of non-coding sequences [26-30]. Only $2 \%$ of the mammalian genome encodes mRNAs, whereas the vast majority of this genome (80\%) transcribe as long and short ncRNAs [26]. ncRNAs form a very heterogeneous group of transcribed RNAs, including long non-coding RNAs, siRNAs, miRNAs, piRNAs, PARs, snoRNAs, xiRNAs, sdRNAs, moRNAs, MSY-RNAs, tel-sRNAs, and crasiRNAs [26]. Previous studies have reported that more than half of all known miRNAs are located in introns of either protein-coding mRNAs or non-coding transcription units, whereas exons of nonprotein-coding transcripts code for only around $10 \%$ of the miRNAs $[31,43]$. In some cases, the miRNAs are encoded in either an exon or an intron, depending on the alternative splicing of the premRNA $[31,43]$. Our results strongly substantiate the fact that exon IDX-rasISS1 may contain a ncRNA and this work also unveils some potential function of this ncRNA. Exon IDX-rasISS1 is hosted on the important oncogene $\mathrm{H}$-Ras pre-mRNA, expressed as a result of the alternative splicing of $\mathrm{H}$-Ras (exclusion of the IDX exon to form p21 H-Ras).

This study was based on three initial observations: a) our previous control experiments revealed that overexpression of the Hairpin-Loop RNA decreased the amount of p68 RNA helicase (see one example in Figure 3 A lanes 3 and 4); b) moreover, the secondary structure illustrated in Figure 1 may indicate that a miRNA could be processed from the Hairpin-Loop and c) the Hairpin-Loop was found to be very stable in native gels and it is conserved in different mammalian cells $[8,14,15]$. Furthermore, other research outcomes claimed that $10-30 \%$ of a spliced intron are exported into the cytoplasm with a moderate half-life $[44,45]$.

Herein, we have further highlighted that the Hairpin-Loop may contain an exon-intron miRNAlike structure targeting the 3'-UTR of the p68 RNA helicase (Figure 2 and 3). Furthermore, it is also significant that the second putative binding site inside the p68 RNA helicase CDS was not as effective as the $3^{\prime}$-UTR sequence (Figure 3B). Indeed, this observation is not rare. As in the case for let-7 versus Dicer, miRNAs can target CDS [46]. However, the proposed model presumes that a high degree of complementarity results in effective targeting of CDS, as is the case with siRNA [47]. Previous studies have confirmed that miRNAs target sites at the $3^{\prime}$-terminus of the transcripts are more likely to be highly evolutionarily conserved and suggested that the proximity of the poly(A) tail and the possibility of an interaction between the RISC complex and the 5' cap mRNA after circularization, is essential to enable the miRNA to inhibit translation $[47,48]$. In agreement with this model, we observed (Figure 2) that IDX can target the 3 '-terminus of 3 '-UTR and that bulge nucleotides may be present immediately after the seed sequence; this observation which corresponds with the model of effective nonconserved sites and siRNA off-targets described by Grimson et al. [49]. Several general mechanisms, including the linearized release of intron D (containing the Hairpin-Loop) by exonucleolytic trimming, the release of the Hairpin-Loop by the action of Drosha or DCL1 inside the commitment complex, and the action of small introns, known as mirtrons, which are released without Drosha enzymatic activity, can liberate the Hairpin-Loop from the spliced intron [50]. Intron sequences are under less stringent selection pressure than exons. The majority of miRNAs are seeded inside introns; it has been proposed that this localization allows a better evolution of the miRNAs [50]. Here we emphasized an miRNA-like sequence on the Hairpin-Loop that combines both a high and less stringent selection pressure. 
It is well known that the presence of dsRNA may activate the interferon-inducible PKR, which, in turn, functions as an important inhibitor of viral invasion by blocking protein synthesis and inducing apoptosis [35, 36]. Impairment of translation initiation mediated by PKR results from phosphorylation of the $\alpha$-subunit of the EIF2 $\alpha$ at Ser 51 [37]. Our findings clearly substantiate the fact that overexpression of the dsRNA hairpin loop fails to activate the phosphorylation of EIF $2 \alpha$, and, therefore, does not activate PKR interferon-induced apoptosis (Figure 4C). Indeed, overexpression of the Hairpin-Loop even reduces the apoptotic peaks, as detected by FACS (Figure 4 and B). Consequently, the Hairpin-Loop is recognized as endogenous RNA rather than as a foreign viral sequence.

We have reported previously that the knock-down of p68 RNA helicase by RNA-mediated interference facilitates the upregulation of endogenous p19 H-Ras, G1/S delay, but no apoptosis [9, 15], and upregulation of several miRNAs (our unpublished results). We have complemented these previous results here by depicting identical consequences of overexpression of the Hairpin-Loop as that of p68 RNA helicase RNAi, including upregulation of p19 (Figure 4C), G1/S delay (Figure 4A and B), no apoptosis (Figure 4A and B), and miR-206 upregulation (Figure 5). Therefore, we can conclude that the effect of Hairpin-Loop overexpression is fundamentally attributed to the downregulation of p68 RNA helicase.

Finally, we have highlighted that our approach to the search for similar Hairpin-Loop sequences, which makes use of an alternative splicing array technique after incubation with cDNAs from cells containing RNA-mediated interference of p68 RNA helicase, allows us to identify similar HairpinLoop structures, also regulated by p68 RNA helicase. This is the case for MAPK12/ERK6, which encompasses a putative Hairpin-Loop containing the alternative exon E8 (Figure 7). MAPK12/ERK6 is engaged in signal transduction during the differentiation of myoblasts into myotubes [42, 51]. It is remarkable to note that $5^{\prime}$ SS is trapped inside the Hairpin-Loop in the predicted secondary structure of MAPK12/ERK6, and this structure should, therefore, forbid U1snRNP binding, as is also the case for the IDX-rasISS1 Hairpin-Loop. All these results validate our observation that p68 RNA helicase is responsible for the upregulation of the Hairpin-Loop, thereby suggesting an inhibitory feedback mechanism for the p68 RNA helicase by higher levels of the Hairpin-Loop. The mechanism of this regulatory ncRNA should be elucidated in future studies to analyze whether the Hairpin-Loop is acting by down-regulation of p68 RNA levels by antisense inhibition, transcriptional degradation, or translational inhibition.

p19 H-Ras downregulation increases cell proliferation [8, 9]. Then, a higher level of IDX HairpinLoop is a consequence of higher alternative splicing toward p $21 \mathrm{H}$-Ras protein, which, in the absence of any inhibitory feedback regulation, would have been able to drive the cell-cycle to higher proliferative cell growth. Therefore, Hairpin-Loop and p68 helicase levels play a physiologic role in regulating some proliferative cancer processes.

\section{Conclusions}

In summary, our research has affirmed that the vast majority of genome sequences are likely to contain many more expressed and functional RNAs than previously expected. The example of IDXrasISS1 or E8 in MAPK12/ERK6 described herein is, therefore, only the tip of the iceberg of a family of putative ncRNAs that are significantly regulated by alternative splicing and dependent on helicase activity. Furthermore, we have documented a rationale to study the function of this group of 
ncRNAs, which are involved in several aspects of cell survival or miRNA expression. Finally, further experimental works are required to elucidate the presence and physiological function of IDX-rasISS1 or E8 in MAPK12/ERK6 and of the relative miRNAs.

\section{Acknowledgments}

We are grateful to Dr. Jaume Comas (Scientific and Technical Services of the University of Barcelona) for his technical assistance with the flow cytometer and to Marta Casado (Institute for Molecular Biology of Barcelona, IBMB-CSIC) for her technical help and discussions regarding the RTPCR analysis. This work was supported by FIS PI08007 and the Fundación Eugenio Rodríguez Pascual. M.K. was a recipient of an FPU (MEC) fellowship. This manuscript was prepared during COVID-19 lockdown.

\section{Author Contributions}

M.K. and M.B.E. proposed conceived and designed the research. M.K. developed the methods and experimental research. M.B.E. designed and performed numerous in silico assays and prepared the manuscript.

\section{Competing Interests}

The authors have declared that no competing interests exist.

\section{References}

1. Barbacid M. Ras genes. Ann Rev Biochem. 1987; 56: 779-827.

2. Malumbres $M$, Pellicer A. RAS pathways to cell cycle control and cell transformation. Front Biosci. 1998; 3: d887-912.

3. DeNicola GM, Tuveson DA. RAS in cellular transformation and senescence. Eur J Cancer. 2009; 45 Suppl 1: 211-216.

4. Gripp KW, Lin AE, Stabley DL, Nicholson L, Scott Cl, Jr., Doyle D, et al. HRAS mutation analysis in Costello syndrome: genotype and phenotype correlation. Am J Med Genet A. 2006; 140: 1-7.

5. Gripp KW, Stabley DL, Nicholson L, Hoffman JD, Sol-Church K. Somatic mosaicism for an HRAS mutation causes Costello syndrome. Am J Med Genet A. 2006; 140: 2163-2169.

6. Gripp KW, Morse LA, Axelrad M, Chatfield KC, Chidekel A, Dobyns W, et al. Costello syndrome: Clinical phenotype, genotype, and management guidelines. Am J Med Genet A. 2019; 179: 1725-1744.

7. Cohen JB, Broz SD, Levinson AD. Expression of the H-ras proto-oncogene is controlled by alternative splicing. Cell. 1989; 58: 461-472.

8. Guil S, De La Iglesia N, Fernandez-Larrea J, Cifuentes D, Ferrer JC, Guinovart JJ, et al. Alternative splicing of the human proto-oncogene $\mathrm{c}-\mathrm{H}$-ras renders a new Ras family protein that trafficks to cytoplasm and nucleus. Cancer Res. 2003; 63: 5178-5187.

9. Camats M, Kokolo M, Heesom KJ, Ladomery M, Bach-Elias M. P19 H-ras induces G1/S phase delay maintaining cells in a reversible quiescence state. PLoS One. 2009; 4: e8513.

10. Pells S, Divjak M, Romanowski P, Impey H, Hawkins NJ, Clarke AR, et al. Developmentallyregulated expression of murine K-ras isoforms. Oncogene. 1997; 15: 1781-1786. 
11. Huang MY, Cohen JB. The alternative H-ras protein $\mathrm{p} 19$ displays properties of a negative regulator of p21Ras. Oncol Res. 1997; 9: 611-621.

12. Jang SM, Kim JW, Kim CH, Kim D, Rhee S, Choi KH. p19ras Represses proliferation of non-small cell lung cancer possibly through interaction with Neuron-Specific Enolase (NSE). Cancer Letters. 2010; 289: 91-98.

13. Garcia-Cruz R, Camats M, Calin GA, Liu CG, Volinia S, Taccioli C, et al. The role of p19 and p21 $\mathrm{H}$-Ras proteins and mutants in miRNA expression in cancer and a Costello syndrome cell model. BMC Med Genet. 2015; 16: 46.

14. Guil S, Gattoni R, Carrascal M, Abián J, Stévenin J, Bach-Elias M. Roles of hnRNP A1, SR proteins, and $\mathrm{p} 68$ helicase in C-H-ras alternative splicing regulation. Mol Cel Biol. 2003; 23: 2927-2941.

15. Camats M, Guil S, Kokolo M, Bach-Elias M. P68 RNA helicase (DDX5) alters activity of cis-and trans-acting factors of the alternative splicing of H-Ras. PLoS ONE. 2008; 3: e2926.

16. Iggo RD, Lane DP. Nuclear protein p68 is an RNA-dependent ATPase. EMBO J. 1989; 8:18271831.

17. Bleichert F, Baserga SJ. The long unwinding road of RNA helicases. Mol Cell. 2007; 27: 339-352.

18. Linder P, Jankowsky E. From unwinding to clamping - the DEAD box RNA helicase family. Nat Rev Mol Cell Biol. 2011; 12: 505-516.

19. Causevic M, Hislop RG, Kernohan NM, Carey FA, Kay RA, Steele RJ, et al. Overexpression and poly-ubiquitylation of the DEAD-box RNA helicase p68 in colorectal tumours. Oncogene. 2001; 20: 7734-7743.

20. Shin S, Rossow KL, Grande JP, Janknecht R. Involvement of RNA helicases p68 and p72 in colon cancer. Cancer Res. 2007; 67: 7572-7578.

21. Clark EL, Coulson A, Dalgliesh C, Rajan P, Nicol SM, Fleming S, et al. The RNA helicase p68 is a novel androgen receptor coactivator involved in splicing and is overexpressed in prostate cancer. Cancer Res. 2008; 68: 7938-7946.

22. Mooney SM, Grande JP, Salisbury JL, Janknecht R. Sumoylation of p68 and p72 RNA helicases affects protein stability and transactivation potential. Biochemistry. 2010; 49: 1-10.

23. Dai TY, Cao L, Yang ZC, Li YS, Tan L, Ran XZ, et al. P68 RNA helicase as a molecular target for cancer therapy. J Exp Clin Cancer Res. 2014; 33: 64.

24. Hashemi V, Masjedi A, Hazhir-Karzar B, Tanomand A, Shotorbani SS, Hojjat-Farsangi M, et al. The role of DEAD-box RNA helicase p68 (DDX5) in the development and treatment of breast cancer. J Cell Physiol. 2019; 234: 5478-5487.

25. Kokolo M, Bach-Elias M. Downregulation of p68 RNA Helicase (DDX5) activates a survival pathway involving mTOR and MDM2 Signals. Folia Biol. 2017; 63: 52-59.

26. Taft RJ, Pang KC, Mercer TR, Dinger M, Mattick JS. Non-coding RNAs: Regulators of disease. J Pathol. 2010; 220: 126-139.

27. Esteller M. Non-coding RNAs in human disease. Nat Rev Genet. 2011; 12: 861-874.

28. Matsui M, Corey DR. Non-coding RNAs as drug targets. Nat Rev Drug Discov. 2017; 16: 167-179.

29. Anastasiadou E, Jacob LS, Slack FJ. Non-coding RNA networks in cancer. Nat Rev Cancer. 2018; 18: 5-18.

30. Abramowicz A, Story MD. The long and short of it: The emerging roles of non-coding RNA in small extracellular vesicles. Cancers. 2020; 12: 1445.

31. Rodriguez A, Griffiths-Jones S, Ashurst JL, Bradley A. Identification of mammalian microRNA host genes and transcription units. Genome Res. 2004; 14: 1902-1910. 
32. Wolfien M, Brauer DL, Bagnacani A, Wolkenhauer O. Workflow Development for the Functional Characterization of ncRNAs. Methods Mol Biol. 2019; 1912: 111-132.

33. Livak KJ, Schmittgen TD. Analysis of relative gene expression data using real-time quantitative PCR and the 2- $\triangle \Delta C T$ Method. Methods. 2001; 25: 402-408.

34. Dignam JD, Lebovitz RM, Roeder RG. Accurate transcription initiation by RNA polymerase II in a soluble extract from isolated mammalian nuclei. Nucleic Acids Res. 1983; 11: 1475-1489.

35. Gale M Jr, Tan SL, Katze MG. Translational control of viral gene expression in eukaryotes. Microbiol Mol Biol Rev. 2000; 64: 239-280.

36. Gal-Ben-Ari S, Barrera I, Ehrlich M, Rosenblum K. PKR: A Kinase to Remember. Front Mol Neurosci. 2019; 11: 480.

37. Dever TE. Gene-specific regulation by general translation factors. Cell. 2002; 108: 545-556.

38. Donnelly N, Gorman AM, Gupta S, Samali A. The EIF2 $\alpha$ kinases: Their structures and functions. Cell Mol Life Sci. 2013; 70: 3493-3511.

39. Tavazoie SF, Alarcón C, Oskarsson T, Padua D, Wang Q, Bos PD, et al. Endogenous human microRNAs that suppress breast cancer metastasis. Nature. 2008; 451: 147-152.

40. Pan JY, Sun CC, Bi ZY, Chen ZL, Li SJ, Li QQ, et al. miR-206/133b Cluster: A Weapon against Lung Cancer? Mol Ther Nucleic Acids. 2017; 8: 442-449.

41. Rayburn ER, Ezell SJ, Zhang R. Recent advances in validating MDM2 as a cancer target. Anticancer Agents Med Chem. 2009; 9: 882-903.

42. Lechner C, Zahalka MA, Giot JF, Møller NP, Ullrich A. ERK6, a mitogen-activated protein kinase involved in C2C12 myoblast differentiation. Proc Natl Acad Sci U S A. 1996; 93: 4355-4359.

43. Hubé $F$, Ulveling $D$, Sureau A, Forveille $S$, Francastel $C$. Short intron-derived ncRNAs. Nucleic Acids Res. 2017; 45: 4768-4781.

44. Clement JQ, Qian L, Kaplinsky N, Wilkinson MF. The stability and fate of a spliced intron from vertebrate cells. RNA. 1999; 5: 206-220.

45. Talhouarne GJ, Gall JG. Lariat intronic RNAs in the cytoplasm of vertebrate cells. Proc Natl Acad Sci USA. 2018; 115: E7970-E7977.

46. Zhang $K$, Zhang $X$, Cai Z, Zhou J, Cao R, Zhao Y, et al. A novel class of microRNA-recognition elements that function only within open reading frames. Nat Struct Mol Biol. 2018; 25: 10191027.

47. Forman JJ, Coller HA. The code within the code: MicroRNAs target coding regions. Cell Cycle. 2010; 9: 1533-1541.

48. Liu G, Zhang R, Xu J, Wu Cl, Lu X. Functional conservation of both CDS-and 3'-UTR-located microRNA binding sites between species. Mol Biol Evol. 2015; 32: 623-628.

49. Grimson A, Farh KK, Johnston WK, Garrett-Engele P, Lim LP, Bartel DP. MicroRNA targeting specificity in mammals: Determinants beyond seed pairing. Mol Cell. 2007; 27: 91-105.

50. Brown JW, Marshall DF, Echeverria M. Intronic noncoding RNAs and splicing. Trends Plant Sci. 2008; 13: 335-342.

51. Goldsmith ZG, Dhanasekaran DN. G protein regulation of MAPK networks. Oncogene. 2007; 26 : 3122-3142. 


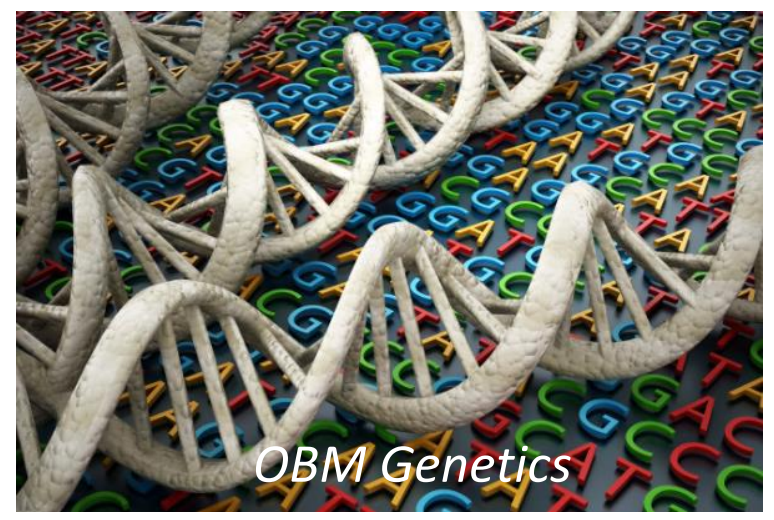

Enjoy OBM Genetics by:

1. Submitting a manuscript

2. Joining in volunteer reviewer bank

3. Joining Editorial Board

4. Guest editing a special issue

For more details, please visit:

http://www.lidsen.com/journals/genetics 